\title{
Learning curve in robotic transabdominal preperitoneal (rTAPP) ventral hernia repair: a cumulative sum (CUSUM) analysis
}

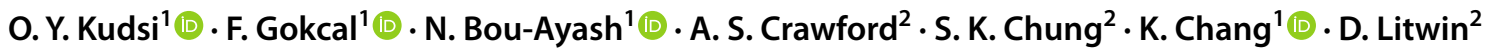

Received: 15 April 2020 / Accepted: 25 May 2020 / Published online: 3 June 2020

(c) Springer-Verlag France SAS, part of Springer Nature 2020

\begin{abstract}
Purpose rTAPP-VHR is a novel technique which may be added to a surgeon's armamentarium. We aim to evaluate the robotic transabdominal preperitoneal ventral hernia repair (rTAPP-VHR) learning curve based on operative times while accounting for peritoneal flap integrity.

Methods We performed a retrospective analysis of a database collected over a 7-year period. Patients with primary ventral hernias were included and a cumulative sum analysis(CUSUM) was used to create learning curves for three subsets of operative times. A risk-adjusted CUSUM (RA-CUSUM) accounted for repair quality based on peritoneal flap completeness. The flap was considered as incomplete when peritoneal gaps were unable to be closed.

Results 105 patients undergoing rTAPP-VHR were included. Learning curves were created for skin-to-skin, console, and off-console times. Patients were divided into three phases. In terms of skin-to-skin times, both phase $2 \& 3$ had a mean 11 min shorter than that of phase 1 ( $p=0.0498, p=0.0245$, respectively), with a steady decrease after forty-six cases. An incomplete peritoneal flap was noted in $25 / 36$ patients in phase 1 , as compared to $5 / 24$ and $5 / 45$ patients in phase $2 \& 3$, respectively. When risk-adjusted for peritoneal flap completeness, gradually decreasing skin-to-skin times were observed after sixty-one cases. In terms of off-console times, the mean across three phases was $14 \mathrm{~min}$, with marked improvement after forty-three cases. Conclusions Forty-six cases were needed to achieve steadily decreasing operative times. We can assume that ensuring goodquality repairs, through maintenance of peritoneal flap integrity, was gradually improved after sixty-one cases. Moreover, familiarization with port placements and robotic docking was accomplished after forty-three cases.
\end{abstract}

Keywords Robotic ventral hernia repair · Learning curve $\cdot$ Transabdominal preperitoneal $\cdot$ TAPP

\section{Introduction}

Ventral hernia repair (VHR) has evolved and ranged from open to minimally invasive techniques with adjuncts including various mesh placements. Laparoscopic ventral hernia repair (LVHR) was initially performed as a bridging intraperitoneal onlay mesh technique (IPOM) in the 1990s [1].

The preliminary results of this paper were presented as an oral presenation in the INSAC-Natural and Health Sciences 2020 congress (Turkey).

\section{O. Y. Kudsi}

omar.kudsi@tufts.edu

1 Department of Surgery, Good Samaritan Medical Center, Tufts University School of Medicine, One Pearl Street, Brockton, MA 02301, USA

2 Department of Surgery, University of Massachusetts Medical School, Worcester, MA, USA
Other mesh positions and techniques gradually arose, such as transabdominal preperitoneal (TAPP) repair, which may minimize bowel-associated complications [2]. Furthermore, early studies suggest that TAPP repair provides comparable outcomes to IPOM repair in both conventional laparoscopy and robotics [3, 4]. Technical requirements in the execution of laparoscopic ventral hernia through an exclusively TAPP approach have made this method less popular. The advent of the robotic platform in hernia repairs has generated immense interest among the surgical community. However, a significant barrier to the adoption of rTAPP-VHR is the learning curve (LC), which has not yet been clearly defined.

In this study, we report the LC of rTAPP-VHR based on operative times, while assessing repair quality. We hope to add our experience to the literature to highlight the technical aspects of rTAPP-VHR and elucidate barriers that surgeons interested in adopting this procedure may encounter. 


\section{Materials and methods}

\section{Patients}

We retrospectively reviewed a prospectively collected database of cases performed between February 2013 and January 2020. Patients who underwent rTAPP repair were selected, while other mesh positions (intraperitoneal, retromuscular) were omitted. To obtain a homogenous study group, patients who underwent rTAPP for elective, midline, and primary hernias were included. Patients who underwent robotic incisional or emergent VHR, as well as patients who had concomitant procedures, were excluded.

\section{Surgeon}

All operations were performed by one surgeon at a single institution, trained in Minimally Invasive Surgery (MIS). The surgeon had completed simulator-assisted training, animal-based training, cadaver training, live case observations, proctoring, and mentorship through various social media platforms [5], prior to initiating robotic surgical practice. Additionally, multiple robotic procedures, including robotic intraperitoneal onlay mesh (rIPOM) VHR, have been conducted leading up to the first rTAPP case included in this study.

\section{Variables}

The variables evaluated include patient demographics, hernia characteristics, operative variables, and postoperative variables.

Patient demographics include the following: age, sex, body mass index (BMI), comorbidities, and risk factors. The hernia characteristics and operative variables studied include hernia size, hernia defect closure, completeness of the peritoneal flap, mesh type and size, method of fixation, procedure duration, estimated blood loss (EBL), conversion to other approaches, and intraoperative complications. Early postoperative results include the hospital length of stay (LOS), hospital readmission within a 30-day postoperative period, and post-op complications.

Hernia dimensions were measured according to the European Hernia Society (EHS) recommendations [6]. Based on intraoperative measurements of hernia defects, as well as mesh dimensions, the variables of the defect area, mesh area, and mesh-to-defect ratio were determined using conventional mathematical formulas as described previously [4]. Operative times were examined as three separate variables including skin-to-skin time, console time and off-console time. Skin-to-skin time was defined as the time from skin incision to skin closure. Console time consists of the surgeon's active surgery duration. The off-console time was calculated by subtracting console time from skin-to-skin time and was used to reflect the time required to place trocars, dock the robot, undock the robot, and close any trocar sites. The LOS (days) was defined as the difference in time between the date of the operation and the date of hospital discharge.

Postoperative complications were reviewed as documented in the surgeon's follow-up visits, as well as the patients' medical records and clinical charts. All complications were categorized according to the Clavien-Dindo classification system [7]. Surgical wound complications were represented in accordance with previously published studies [8]. Surgical site events (SSEs) were further classified as surgical site infections (SSIs-superficial, deep and organ-space infections), surgical site occurrences (SSOs-including fluid collections such as seroma and hematoma), and surgical site occurrence or infection procedural interventions ( $\mathrm{SSO}$ / SSI-PIs; SSOs or SSIs requiring any procedural intervention such as reopening a wound, placing a drain, percutaneous aspiration, or reoperation). Postoperative morbidity score was measured using the Comprehensive Complication Index (CCI®, University of Zurich, Zurich, Switzerland) [9]. The Morales-Conde classification algorithm was used to describe the severity of a seroma complication [10].

\section{Surgical technique}

The operation was performed under general anesthesia with the patient placed in the supine position. A Veress needle was placed two fingerbreadths below the costal margin in the left upper quadrant to establish pneumoperitoneum with a pressure of $15 \mathrm{mmHg}$. Two working ports and a single camera port were used. Based on the hernia defect size, the appropriate mesh size was chosen to obtain the necessary overlap. Using monopolar scissors and a bipolar grasper, the peritoneum on the side ipsilateral to the trocars was grasped and cut to enter the preperitoneal space at least $5 \mathrm{~cm}$ away from the defect. Preperitoneal dissection was extended at least $5 \mathrm{~cm}$ circumferentially around the defect to provide adequate mesh overlap. The intraabdominal pressure was then reduced (6-8 $\mathrm{mmHg}$ ). Primary closure of the hernia defect was achieved by running a long-lasting absorbable barbed suture (Stratafix $0^{\mathrm{TM}}$ on CT-1 needle, Ethicon, Somerville, NJ, USA), taking 5-8 mm bites of fascia every $5 \mathrm{~mm}$ in a running fashion. The mesh was secured to the posterior fascia with an absorbable suture. In case of failure to maintain an intact peritoneal flap, coated meshes were used. Otherwise, uncoated meshes were used. If present, small tears in the peritoneum were repaired using absorbable sutures. The peritoneal flap was closed with a barbed absorbable suture (2-0 V-Loc ${ }^{\mathrm{TM}}$; Medtronic, Minneapolis, 
MN, USA). Dehiscence was assessed by increasing intraabdominal pressure to $15 \mathrm{mmHg}$. The patient-side cart was then undocked. The trocars were removed with the release of pneumoperitoneum. The fascia for trocar sites $10 \mathrm{~mm}$ or larger was sutured to decrease the risk of future incisional hernias. Long-acting local anesthetic was injected in the incisions for postoperative pain management [11].

\section{Outcome measurement}

One measure of the surgical process is the success or completion rate of a certain procedural step [12]. For the rTAPP procedure, one important step is the development of an intact peritoneal flap. The flap is considered as incomplete when peritoneal gaps were unable to be closed using sutures, and instead required bridging with a coated mesh. The primary outcome of this study was operative times, whilst evaluating peritoneal flap integrity as a secondary outcome.

\section{Statistical analysis}

Statistical analysis was performed using the Statistical Analysis System program (SAS version 9.4) and SPSS software (Statistical Package for Social Sciences for Windows Version 22). Additional graphical illustrations were prepared using Microsoft Excel 2019 (Microsoft Office for Windows, Redmond, Washington, DC, USA).

\section{Cumulative sum (CUSUM) analysis}

LCs were separately assessed for skin-to-skin time, console time, and off-console time. To detect change in the surgeon's performance for these time spans, CUSUM curves of each time spans were calculated based on the variable's sample mean. Inflection points were indicated at each set of three or more consecutive negative values [13]; these inflection points were used to divide the patients into separate phases. A linear regression model was then fit to match each CUSUM curve.

\section{Risk-adjusted CUSUM (RA-CUSUM) analysis}

Risk-adjusted CUSUM (RA-CUSUM) analysis was also used to depict success or failure in peritoneal dissection during the LC period. Risk adjustment was obtained by fitting a logistic regression model predicting failure of the peritoneal dissection using forward selection, with variables significant $p<0.20$ in univariate association considered for inclusion. The predicted probabilities from this model were outputted and used to risk adjust as follows. The RA-CUSUM starts at value 0 . For each failure (incomplete peritoneal flap) the value is incremented by $(1$ - predicted probability of failure). For each success (complete peritoneal flap or repaired small tear) the value is decreased by the predicted probability of failure. Patients were again grouped into phases by inflection points.

\section{Comparative analysis}

Once patients were grouped the groups were compared. Chi-square test or Fisher's exact test were used for categorical variables. One-way ANOVA test or Kruskal-Wallis test were used for continuous variables as appropriate. Categorical variables are presented in terms of frequency ( $n$ and/or \%), while continuous variables were reported as the mean \pm the standard deviation (SD) for normal distributions or the median with interquartile range (IQR) for non-normal distributions. A $p$-value of $<0.05$ was considered as statistically significant.

\section{Results}

In our cohort of 598 patients who underwent RVHR, 105 patients with midline, primary ventral, and elective rTAPP repairs were identified. The distribution of operative times (skin-to-skin, console, and off-console) among consecutive cases is shown in Fig. 1. The corresponding transition points were determined using a CUSUM analysis.

The mean skin-to-skin time among these 105 patients was 54.84 min. Using the CUSUM method, a LC was obtained showing cumulative differences from the mean. Subsequently, patients were grouped into three phases (Online Resource Fig. 1a). The best fit quadratic line for the LC has an r-square value of 0.908 (Fig. 2a), with the corresponding function:

Skin-to-skin time $=123.75-18.56(\mathrm{case}$ number $)+1.34\left(\right.$ case number $\left.{ }^{2}\right)-0.028($ case number $\left.{ }^{3}\right)+0.00021\left(\right.$ case number $\left.{ }^{4}\right)-0.0000005($ case number $\left.{ }^{5}\right)$. The maximum value of this function is at approximately case number 46 .

ANOVA was used to test skin-to-skin time with the abovementioned categorization. The three phases were found to have significantly different mean skin-to-skin times $(p=0.048)$ (Online Resource Fig. 1b).

The mean console time among these 105 patients is $40.85 \mathrm{~min}$. A LC was obtained using similar methods (Online Resource Fig. 2a). The best fit quadratic line has an $r$-square value of 0.925 (Fig. 2b), with the corresponding function:

Console time $=117.87-22.73$ (case number $)+1.41$ (case number $\left.{ }^{2}\right)-0.027\left(\right.$ case number $\left.{ }^{3}\right)+0.000198($ case number $\left.{ }^{4}\right)-0.0000005\left(\right.$ case number $\left.{ }^{5}\right)$. The maximum value of this function is at approximately case number 50 . The mean console time for the three phases was significantly different ( $p=0.012$ ) (Online Resource Fig. 2b). 




Fig. 1 The distribution of skin-to-skin, console, and off-console times among 105 consecutive robotic transabdominal preperitoneal repair (rTAPP)

The mean off-console time among these 105 patients is $13.99 \mathrm{~min}$. Likewise, a LC graph was obtained (Online Resource Fig. 3a). The best fit quadratic line has an $r$-square value of 0.851 (Fig. 2c), with the corresponding function:

Off-console time $=-7.06+6.66$ (case number) $-0.18\left(\right.$ case number $\left.^{2}\right)+0.0012\left(\right.$ case number $\left.^{3}\right)$. The maximum value of this function is at approximately case number 23. The mean off-console time was significantly different across groups ( $p=0.009)$ (Online Resource Fig. 3b).

To provide a measure of quality for these operations, a separate RA-CUSUM analysis was conducted. To do this, a logistic regression model was used to predict the completeness of the peritoneal flap. Univariate analysis showed mesh size $\left(\mathrm{cm}^{2}\right)$ to be the strongest predictor of the lack of peritoneal completeness, so the logistic regression model used was incomplete peritoneal flap (yes/no) $=$ mesh size $\left(\mathrm{cm}^{2}\right)$. The predicted probabilities from this model were outputted and used for risk adjustment (Fig. 3).

A comparison of patient demographics between the three groups, as determined by the RA-CUSUM, is shown in Table 1. There were no differences between the groups in terms of patient characteristics apart from ASA score distribution. A comparison between hernia characteristics and operative variables of the three groups is shown in Table 2 . Although hernia defect size, in $\mathrm{cm}^{2}$, was largest in phase 1, it did not differ significantly between groups. The median mesh size, in $\mathrm{cm}^{2}$, was significantly larger in both phase 2 and-3, as compared to phase 1 . Accordingly, there was a significant difference in mesh-to-defect (M/D) ratio. Polyester based meshes were the most frequently used mesh materials across all phases, and used exclusively in phase 1 . The expanded polytetrafluoroethylene mesh was used in one patient in phase 2 and phase 3. Polypropylene meshes were only used in patients in phase 3. The meshes were fixated in all operations; though minimal suture fixation was performed to secure the mesh for operations in which self-fixating meshes were preferred. No drain placements were observed throughout the entire cohort. A gastric serosal injury occurred in one patient in phase 2 and was simply sutured. There was no conversion to other approaches.

All patients were discharged from the hospital on the same day of procedures except for $1(2.8 \%)$ patient in phase 1 who stayed overnight $(p=0.380)$. One $(2.8 \%)$ patient in phase 1 required hospital readmission within 30-days after discharge due to small bowel obstruction which was managed conservatively $(p=0.380)$. None of the patients experienced a hernia recurrence within the study's 90-day follow-up period. No statistically significant differences in terms of postoperative complications [Clavien-Dindo grades (CD), Comprehensive Complication Index Scores (CCI), and Surgical Site Events (SSE)] were noted among the three groups (Table 3).

\section{Discussion}

LCs provide an introspective view of one's own performance and help us understand the variables affecting proficiency. Equally important is their role in sharing the 
a

Cumulative difference for mean skin-to-skin time

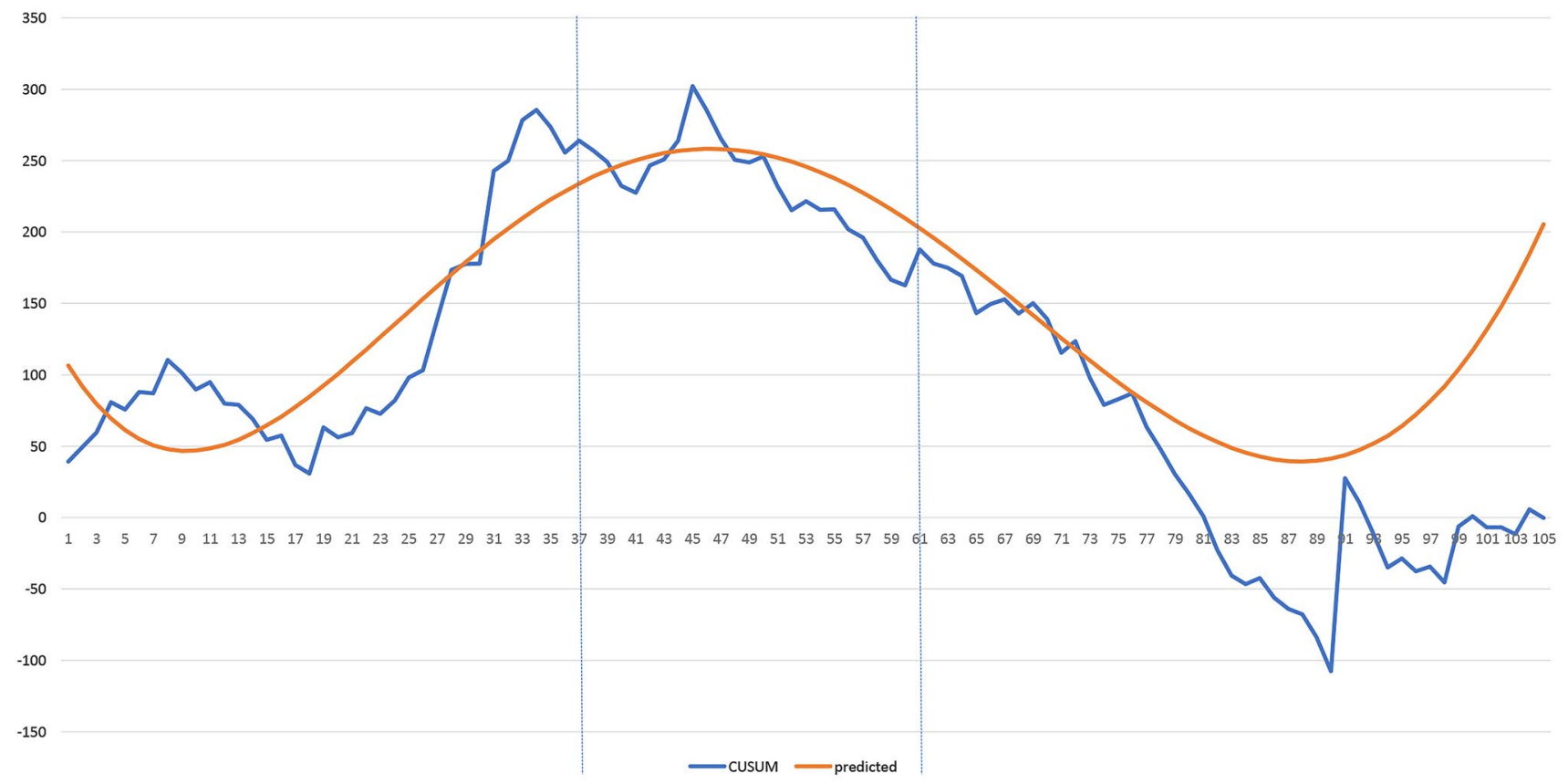

b

Cumulative difference for mean console time

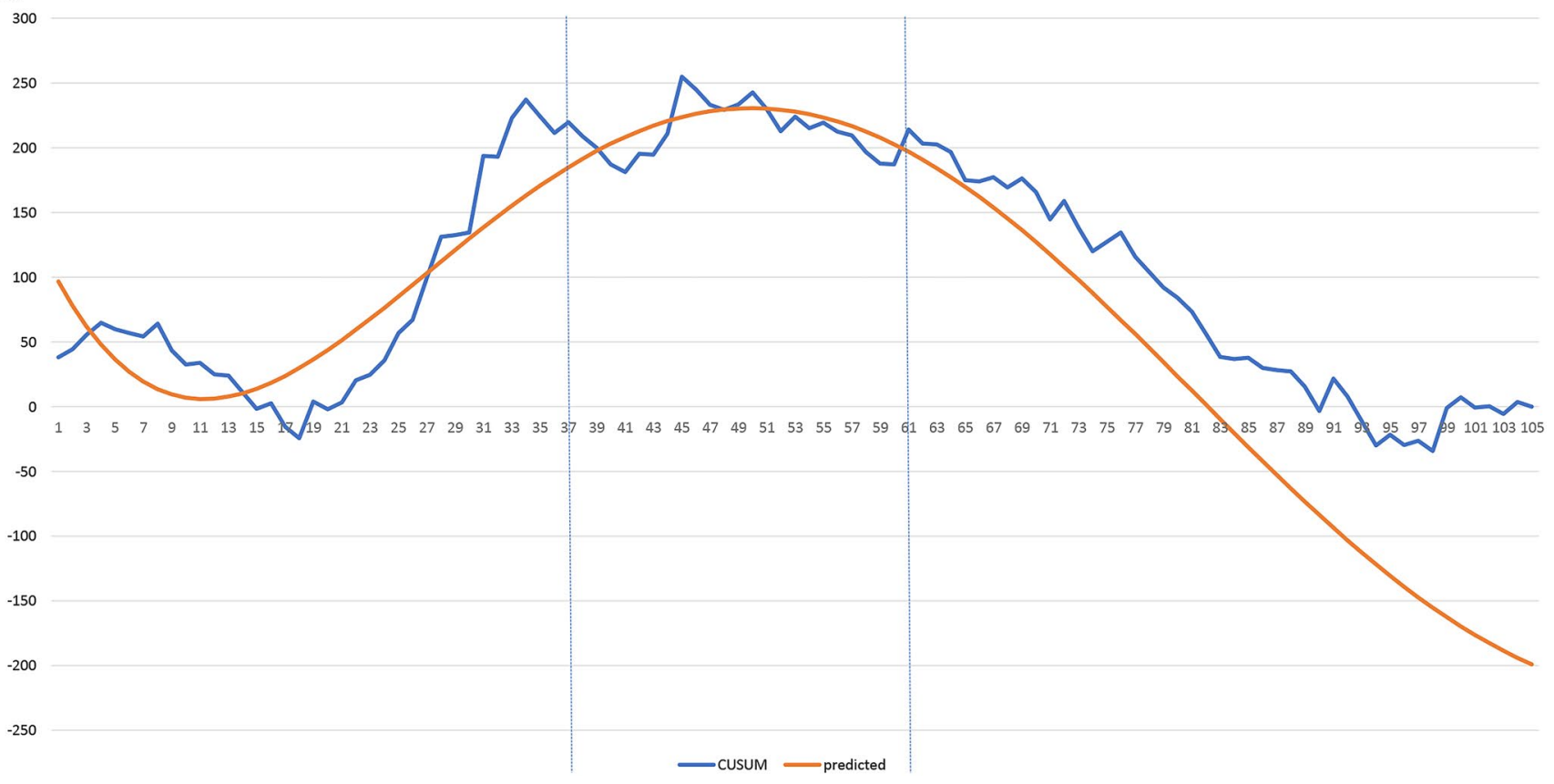

Fig. 2 CUSUM (cumulative sum) analysis of operative times for 105 rTAPP repairs. The $X$ axis indicates consecutive cases, and the $Y$ axis indicates the CUSUM score for skin-to-skin time. While an uphill slope indicates an increasing trend, a downhill slope indicates a decreasing trend. Vertical lines refer to the turning points at which the surgeon transitions from one phase to another. For skin-to-skin time, the best fit quadratic line for the LC has an r-square value of 0.908 .
The maximum value of the corresponding function is at approximately case number 46 (a). For console time, the best fit quadratic line for the LC has an r-square value of 0.925 . The maximum value of the corresponding function is at approximately case number 50 (b). For off-console time, the best fit quadratic line for the LC has an $\mathrm{r}$-square value of 0.851 . The maximum value of the corresponding function is at approximately case number 23 (c) 


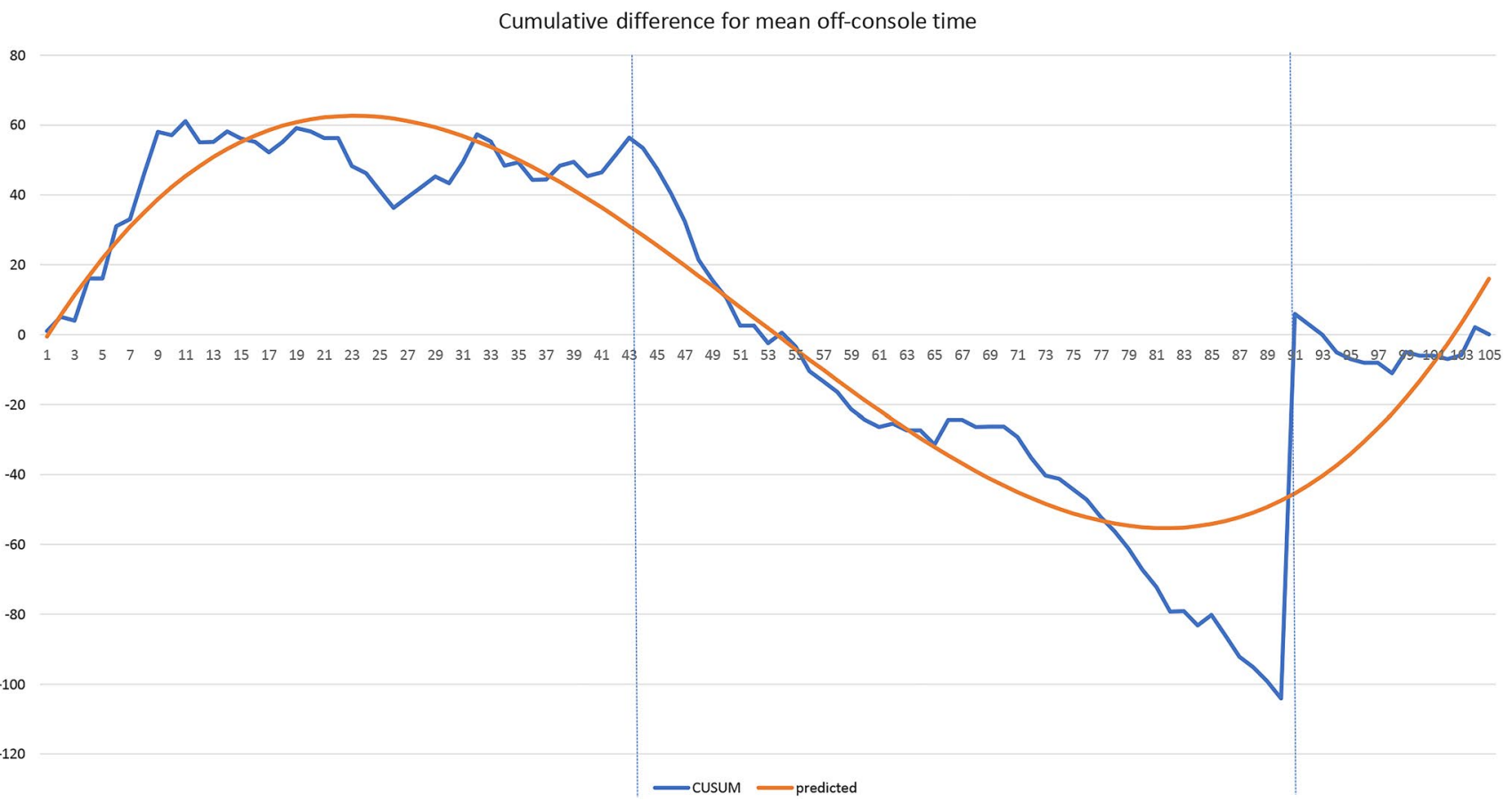

Fig. 2 (continued)



Fig. 3 Risk-adjusted cumulative sum (RA-CUSUM) analysis; The CUSUM starts at value 0 . For each failure (incomplete peritoneal flap) the value is incremented by $(1-$ predicted probability of failure). For each success (complete peritoneal flap) the value is decreased by the predicted probability of incomplete peritoneal flap. In this manner, a RA-CUSUM graph for peritoneal completeness was generated as is displayed above. Phase 1,2, and 3 divisions from the skin-to-skin time CUSUM are shown as vertical reference lines 
Table 1 Patient demographics

\begin{tabular}{|c|c|c|c|c|c|}
\hline & Total $(n=105)$ & Phase $1(n=36)$ & Phase $2(n=24)$ & Phase $3(n=45)$ & $p$ \\
\hline Age, mean \pm SD & $50.1 \pm 14.3$ & $48.6 \pm 13.9$ & $47.5 \pm 12.2$ & $52.8 \pm 15.4$ & 0.257 \\
\hline Sex, female, $n(\%)$ & $30(28.6)$ & $11(30.6)$ & $6(25)$ & $13(28.9)$ & 0.895 \\
\hline BMI, $\mathrm{kg} / \mathrm{m}^{2}$, mean $\pm \mathrm{SD}$ & $31.4 \pm 5.6$ & $31.4 \pm 5.3$ & $31.5 \pm 4.2$ & $31.5 \pm 6.5$ & 0.994 \\
\hline $\begin{array}{l}\text { ASA score } \\
\text { ASA-1, } n(\%) \\
\text { ASA-2, } n(\%) \\
\text { ASA-3, } n(\%)\end{array}$ & $\begin{array}{l}14(13.3) \\
56(53.3) \\
35(33.3)\end{array}$ & $\begin{array}{l}5(13.9) \\
25(69.4) \\
6(16.7)\end{array}$ & $\begin{array}{l}3(12.5) \\
15(62.5) \\
6(25)\end{array}$ & $\begin{array}{l}6(13.3) \\
16(35.6) \\
23(51.1)\end{array}$ & 0.013 \\
\hline $\begin{array}{l}\text { Comorbidities and risk } \\
\text { factors }\end{array}$ & $\begin{array}{l}46(43.8) \\
3(2.9)\end{array}$ & $\begin{array}{l}16(44.4) \\
2(5.6)\end{array}$ & $\begin{array}{l}9(37.5) \\
1(4.2)\end{array}$ & $\begin{array}{l}21(46.7) \\
0(0)\end{array}$ & $\begin{array}{l}0.762 \\
0.299\end{array}$ \\
\hline Hypertension, yes, $n(\%)$ & $9(8.6)$ & $2(5.6)$ & $0(0)$ & $7(15.6)$ & 0.065 \\
\hline $\begin{array}{l}\text { Coronary artery disease, } \\
\text { yes, } n(\%)\end{array}$ & $\begin{array}{l}26(24.8) \\
14(13.3)\end{array}$ & $\begin{array}{l}13(36.1) \\
2(5.6)\end{array}$ & $\begin{array}{l}5(20.8) \\
4(16.7)\end{array}$ & $\begin{array}{l}8(17.8) \\
8(17.8)\end{array}$ & $\begin{array}{l}0.145 \\
0.236\end{array}$ \\
\hline COPD, yes, $n(\%)$ & $2(1.9)$ & $2(5.6)$ & $0(0)$ & $0(0)$ & 0.142 \\
\hline $\begin{array}{l}\text { Smoking, yes, } n(\%) \\
\text { Diabetes mellitus, yes, } \\
n(\%)\end{array}$ & $1(1)$ & $0(0)$ & $0(0)$ & $1(2.2)$ & 0.510 \\
\hline $\begin{array}{l}\text { Immunosuppression, } \\
\text { yes, } n(\%)\end{array}$ & & & & & \\
\hline $\begin{array}{l}\text { History of wound infec- } \\
\text { tion, yes, } n(\%)\end{array}$ & & & & & \\
\hline
\end{tabular}

$S D$ standard deviation, $B M I$ body mass index, $A S A$ the American society of anesthesiologists, $C O P D$ chronic obstructive pulmonary disease

Table 2 Hernia characteristics and operative variables

\begin{tabular}{|c|c|c|c|c|c|}
\hline & Total $(n=105)$ & Phase $1(n=36)$ & Phase $2(n=24)$ & Phase $3(n=45)$ & $p$ \\
\hline Incomplete peritoneal flap, yes, $n(\%)$ & $35(33.3)$ & $25(69.4)$ & $5(20.8)$ & $5(11.1)$ & $<0.001$ \\
\hline Primary defect closure, $n(\%)$ & $101(96.2)$ & $35(97.2)$ & $24(100)$ & $42(93.3)$ & 0.357 \\
\hline Hernia width, cm, median (IQR) & $2(2-2.5)$ & $2(2-3)$ & $2(2-2)$ & $2(2-2.5)$ & 0.345 \\
\hline Hernia defect area, $\mathrm{cm}^{2}$, median (IQR) & $3.1(3.1-7)$ & $4.7(3.1-7.4)$ & $3.1(3.1-4.3)$ & $3.1(3.1-4.9)$ & 0.107 \\
\hline Mesh area, $\mathrm{cm}^{2}$, median (IQR) & $113(63.6-159)$ & $63.6(63.6-113)$ & $144(113-174)$ & $144(113-180)$ & $<0.001$ \\
\hline Mesh/Defect ratio, median (IQR) & $19.2(15-35)$ & $14.5(8-19.2)$ & $35(19.2-35)$ & $24(19.2-35)$ & $<0.001$ \\
\hline $\begin{array}{l}\text { Mesh materials } \\
\text { Polypropylene, } n(\%) \\
\text { Polyester, } n(\%) \\
\text { ePTFE, } n(\%)\end{array}$ & $\begin{array}{l}11(10.5) \\
92(87.6) \\
2(1.9)\end{array}$ & $\begin{array}{l}0(0) \\
36(100) \\
0(0)\end{array}$ & $\begin{array}{l}0(0) \\
23(95.8) \\
1(4.2)\end{array}$ & $\begin{array}{l}11(24.4) \\
33(73.3) \\
1(2.2)\end{array}$ & 0.001 \\
\hline $\begin{array}{l}\text { Mesh fixation } \\
\text { Minimal/self-fixation, } n(\%) \\
\text { Circumferential fixation, } n(\%)\end{array}$ & $\begin{array}{l}26(24.8) \\
79(75.2)\end{array}$ & $\begin{array}{l}11(30.6) \\
25(69.4)\end{array}$ & $\begin{array}{l}9(37.5) \\
15(62.5)\end{array}$ & $\begin{array}{l}6(13.3) \\
39(86.7)\end{array}$ & 0.053 \\
\hline Console time, min., mean \pm SD & $40.8 \pm 15.3$ & $46.7 \pm 17.6$ & $39.8 \pm 13.5$ & $36.7 \pm 13$ & 0.012 \\
\hline Skin-to-skin time, min., mean \pm SD & $54.8 \pm 21.4$ & $61.9 \pm 18.7$ & $50.7 \pm 14$ & $51.2 \pm 25.3$ & 0.048 \\
\hline Estimated blood loss, mL, median (IQR) & $5(5-5)$ & $5(5-5)$ & $5(5-5)$ & $5(5-5)$ & 0.064 \\
\hline
\end{tabular}

ePTFE expanded polytetrafluoroethylene, $I Q R$ interquartile range, $S D$ standard deviation

experience with others in the same medical field, ultimately improving patient care and quality of life [14]. Some studies have defined the LC of LVHR based on operative timing [15], while other studies have measured the LC based on outcomes [16]. Moreover, the recognized interest and importance of studying the LC is universal, as evidenced by the growing number of papers reporting various robotic techniques and associated outcomes [17]. Currently, studies surrounding the LC of RVHR are scarce.
Muysoms et al. produced a LC evaluating operative times and outcomes of robotic transabdominal retromuscular umbilical hernia repair [18]. Patients were divided into tertiles, and using a linear regression model to describe operative times versus the LC across 41 patients, they found a decrease from $126 \mathrm{~min}$ in the first tertile to $102 \mathrm{~min}$ for the third tertile, with significant improvement in retrorectus dissection time. Our study is the first to develop a LC for rTAPP-VHR. We chose to use the CUSUM method due 
Table 3 Postoperative complications

\begin{tabular}{|c|c|c|c|c|c|}
\hline & Total $(n=105)$ & Phase $1(n=36)$ & Phase $2(n=24)$ & Phase $3(n=45)$ & $p$ \\
\hline Any postoperative complication & $6(5.7)$ & $3(8.3)$ & $2(8.3)$ & $1(2.2)$ & 0.410 \\
\hline $\begin{array}{l}\text { Minor complications, yes, } n(\%) \\
\text { Clavien-Dindo Grade-1 } \\
\text { Clavien-Dindo Grade-2 } \\
\text { Major complications, yes, } n(\%) \\
\text { Clavien-Dindo Grade-3a } \\
\text { Grade-3b }\end{array}$ & $\begin{array}{l}3(2.9) \\
1(1) \\
1(1) \\
1(1)\end{array}$ & $\begin{array}{l}1(2.8) \\
1(2.8) \\
1(2.8) \\
0(0)\end{array}$ & $\begin{array}{l}1(4.2) \\
0(0) \\
0(0) \\
1(4.2)\end{array}$ & $\begin{array}{l}1(2.2) \\
0(0) \\
0(0) \\
0(0)\end{array}$ & 0.482 \\
\hline CCI ${ }^{\circledR}$ score, median (range) & $0(0-33.7)$ & $0(0-26.2)$ & $0(0-33.7)$ & $0(0-8.7)$ & 0.397 \\
\hline $\begin{array}{l}\mathrm{SSE}^{*}, n(\%) \\
\mathrm{SSI}, \text { superficial, } n(\%) \\
\mathrm{SSO}, \text { seroma, } n(\%)\end{array}$ & $\begin{array}{l}1(1) \\
1(1) \\
1(1)\end{array}$ & $\begin{array}{l}1(2.8) \\
1(2.8) \\
1(2.8)\end{array}$ & $\begin{array}{l}0(0) \\
0(0) \\
0(0)\end{array}$ & $\begin{array}{l}0(0) \\
0(0) \\
0(0)\end{array}$ & 0.380 \\
\hline SSO/SSI-PI, $n(\%)$ & $0(0)$ & $0(0)$ & $0(0)$ & $0(0)$ & NA \\
\hline
\end{tabular}

CCI comprehensive complication index ${ }^{\circledR}$ (University of Zurich, Zurich, Switzerland), SSEs surgical site events, SSI surgical site infection, SSO surgical site occurrences, SSO/SSI-PI surgical site occurrence or surgical site infection procedural intervention

* Seroma occurred in one patient and became infected in subsequent follow-up, Morale-Conde grade 3E [11]

to its benefits with sequentially analyzing an individual's performance [12].

One of the key operative steps during rTAPP-VHR is the creation of a peritoneal pocket which accommodates mesh placement. The size of the peritoneal flap is comparable to the size of the mesh being placed, and so, larger flaps allow for the use of larger meshes, which result in increased M/D ratio [19]. However, when enlarging the flap during lateral dissection, it becomes increasingly difficult to separate the peritoneum from the overlying fascia due to close adherence of these layers towards the lateral aspect of the rectus sheath. Consequently, large gaps may form in the peritoneum, which cannot be repaired with sutures. In such cases, surgeons may use a coated mesh to bridge these gaps and avoid future intra-abdominal adhesions. A regression model determined mesh area as the strongest predictor for peritoneal flap completeness. In phase 1 of the study, an incomplete peritoneal flap was noted in 25 patients, as compared to 5 patients in phase 2 , and 5 patients in phase $3(p<0.001)$. Consequently, our LC was risk-adjusted for peritoneal flap completeness, revealing a steady decrease in the slope of the graph after the second phase (Fig. 3). We can infer that approximately 61 cases were needed to gain the experience required to achieve good quality repairs, within a considerable time.

As experience is accumulated, dissection of the preperitoneal plane could be accomplished with increased precision to preserve peritoneal integrity. Although the robotic platform provides stability, the lack of haptic feedback may hinder precise dissection of the peritoneal flap and lead to inadvertent tears. Surgeons are pushed towards reliance on visual cues, and with time gain the necessary experience for these procedures. In our study, the mean skin-to-skin time was approximately $55 \mathrm{~min}$. Phases -2 and -3 have mean duration approximately $11 \mathrm{~min}$ shorter than phase $1(p=0.0498$, $p=0.0245$, respectively). While performing preperitoneal dissection, it may be important to highlight the distribution of the preperitoneal adipose layer (also known as fascia propria) [20], which separates the transversalis fascia from the peritoneum and shows variable thickness in different anatomical locations, with abundance at the midline and inferolateral abdominal walls, as demonstrated in Fig. 4. Therefore, it may be beneficial to initiate preperitoneal dissection at the falciform ligament, where the separation of the peritoneum from its overlying layers is facilitated. The dissection can then proceed laterally, using the initial incision as a lead point which guides the appropriate dissection plane.

A closer analysis of the different aspects of rTAPP-VHR is essential to gain a holistic understanding of the LC. Offconsole time reflects the time required to place trocars, dock and un-dock the robot, and close trocar sites. In terms of port placement, trocars placed in a lateral configuration (Fig. 4a) could hinder a surgeon's mobility when dissecting in the clockwise direction due to anatomical boundaries. An oblique trocar configuration (Fig. 4b) could offer surgeons more space and dexterity with dissection. Throughout the study, experience with port placement evolved to accommodate patient body habitus to avoid robotic arm collisions and achieve better mobility. Subsequently, there was a gradual decrease in off-console times after the first phase, apart from a drastic spike for one case in the third phase, whereby a needle was unaccounted for and resulted in significantly prolonged off-console time. This is a testament to the individualized experience of surgeons as well as a combined team effort including scrub nurses, OR technicians, and anesthesiologists.

Reflecting upon the results of this study, we are able to note key factors driving progression throughout this experience. Using the peritoneal pocket as an advancement flap could enable closure of peritoneal gaps by increasing tissue redundancy. Moreover, it also allows for ipsilateral coverage 


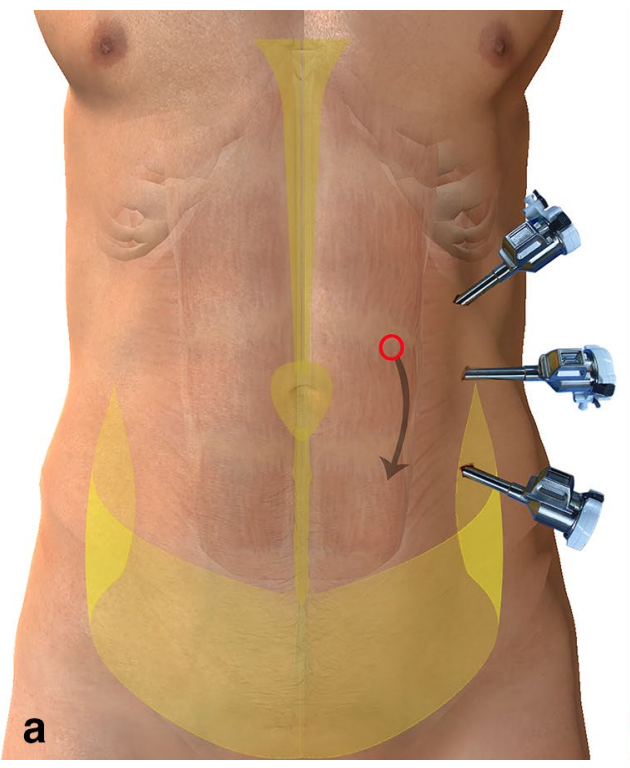

Fig. 4 Illustrations for the distribution of preperitoneal adipose tissue and port configurations. Anchor-shaped shaded areas depict preperitoneal adipose tissue, circles depict the initial peritoneal incision point, arrows depict the direction of the preperitoneal dissection. a Initiation of preperitoneal plane dissection near the linea semilunaris may be difficult due to tight adherence of layers. Furthermore, any port placed below the level of the umbilicus near the anterior superior

of the mesh. Another essential issue arises with dissection at the lateral aspect of the rectus sheath, as it becomes increasingly challenging to separate the peritoneum from the overlying fascia secondary to decreased preperitoneal fatty tissue. Therefore, at that point, it may be beneficial to resume dissection through the pre-transversalis plane instead, effectively avoiding the compromise of peritoneal integrity. To note, the abovementioned techniques are mainly applicable to primary ventral hernias. Regarding incisional hernias, the anatomy of the abdominal wall may be distorted by the previous compromise of virgin tissues, and so, other technical approaches could better suit such cases. A separate LC for incisional VHR could better clarify these issues.

CUSUM learning curves can evaluate the acquisition of new skills and the evolution of those skills as experience is accumulated. Whereas other learning curve methods need to control for different levels of experience, the CUSUM method portrays experience as a continuous variable and provides an objective measure of performance over time [21]. Our study's results represent the initial and continued experience of a single surgeon performing rTAPP-VHR, whose background involves extensive practice with minimally invasive hernia repair and with the robotic platform in general. Therefore, this study's results are mostly applicable to other surgeons with similar backgrounds attempting rTAPP-VHR. An aspect of the CUSUM method which makes it relevant for all levels of experience is that it relies

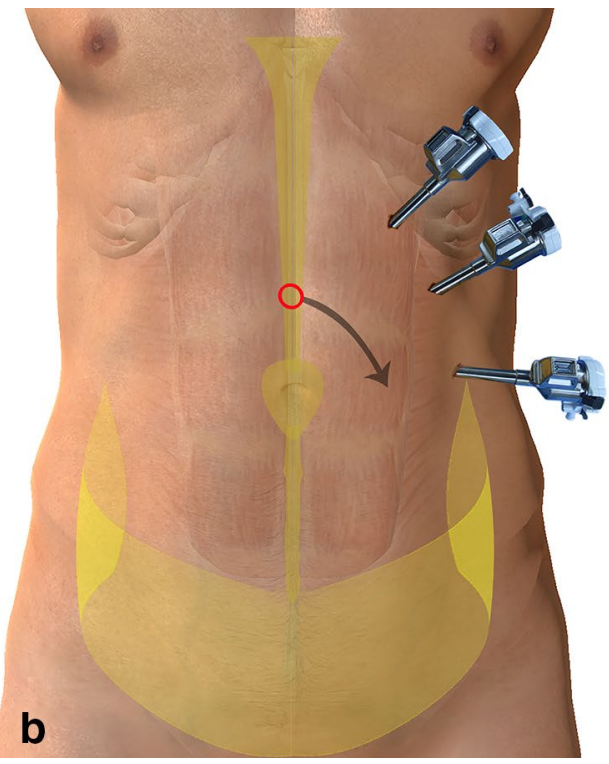

iliac spine (ASIS) while working to repair a centrally located hernia, often results in robotic arm collision. b ' $C$ ' shape port setup at the left upper quadrant provides a distance between the ASIS and the most caudally placed trocar. Initiation of preperitoneal dissection at the midline (above the falciform ligament) may facilitate the recognition of the appropriate dissection plane

on acceptable and unacceptable rates for the selected outcome measure, based on established standards of practice. Unfortunately, data on rTAPP-VHR is limited and learning curves with defined outcome rates for this technique are non-existent. We hope that the increased adoption of rTAPP-VHR and the availability of relevant literature will add more value to this LC and help guide others who wish to implement this technique.

Limitations of this study include its generalizability to surgeons of different backgrounds and expertise. As clarified in our methodology, the surgeon performing these robotic repairs has previous experience with LVHR as well as with the robotic platform for a variety of procedures such as rIPOM VHR. Furthermore, the off-console time LC does not reveal improvement across the three study groups due to a lost needle in one case during phase 3 , as mentioned previously. However, we elected to present this data as a reflection of real-life experience with the robotic platform. Another limitation of this study could be the absence of data surrounding patient abdominal dimensions, as it may play a role in determining the space afforded to complete these procedures efficiently. Detailing operative times for separate surgical steps, as well as providing LCs assessing team performance, may improve our understanding of the factors influencing the success of rTAPP-VHR. Other limitations include the lack of patient-reported outcomes and long-term follow-up necessary for evaluating repair durability. 
This study provides learning curves for rTAPP-VHR using operative times across three different phases. Approximately 61 cases were needed to achieve steadily decreasing operative times while maintaining peritoneal integrity. A separate LC for off-console times suggests that approximately 43 cases were needed to gradually master port placements and robotic docking. Further studies evaluating additional variables such as times for separate surgical stages and long-term outcomes are warranted.

Funding Dr. A has received a teaching course and/or consultancy fees from Intuitive Surgical and W.L. Gore outside the submitted work. No funding has been received for this study.

\section{Compliance with ethical standards}

Conflict of interest Drs. B, C, D, E, F, and G have no conflicts of interest or financial ties to disclose.

Ethical approval The database used for this study approved by the Institutional Review Board.

Human and animal rights All procedures performed in studies involving human participants were in accordance with the ethical standards of the institutional and/or national research committee and with the 1964 Helsinki declaration and its later amendments or comparable ethical standards.

Informed consent Informed consent was obtained from all individual participants included in the study.

\section{References}

1. LeBlanc KA, Booth WV (1993) Laparoscopic repair of incisional abdominal hernias using expanded polytetrafluoroethylene: preliminary findings. Surg Laparosc Endosc 3(1):39-41

2. Ballecer C, Weir A (2017) Robotic transabdominal preperitoneal (rTAPP) hernia repair for ventral hernias. In: Textbook of hernia. Springer, pp 263-272

3. Prasad P, Tantia O, Patle NM, Khanna S, Sen B (2011) Laparoscopic ventral hernia repair: a comparative study of transabdominal preperitoneal versus intraperitoneal onlay mesh repair. J Laparoendosc Adv Surg Tech A 21(6):477-483. https://doi. org/10.1089/lap.2010.0572

4. Gokcal F, Morrison S, Kudsi OY (2019) Short-term comparison between preperitoneal and intraperitoneal onlay mesh placement in robotic ventral hernia repair. Hernia 23(5):957-967. https://doi. org/10.1007/s10029-019-01946-4

5. Myers CG, Kudsi OY, Ghaferi AA (2018) Social media as a platform for surgical learning: use and engagement patterns among robotic surgeons. Ann Surg 267(2):233-235. https://doi. org/10.1097/SLA.0000000000002479

6. Muysoms FE, Miserez M, Berrevoet F, Campanelli G, Champault GG, Chelala E, Dietz UA, Eker HH, El Nakadi I, Hauters P, Hidalgo Pascual M, Hoeferlin A, Klinge U, Montgomery A, Simmermacher RK, Simons MP, Smietanski M, Sommeling C,
Tollens T, Vierendeels T, Kingsnorth A (2009) Classification of primary and incisional abdominal wall hernias. Hernia 13(4):407414. https://doi.org/10.1007/s10029-009-0518-x

7. Dindo D, Demartines N, Clavien PA (2004) Classification of surgical complications: a new proposal with evaluation in a cohort of 6336 patients and results of a survey. Ann Surg 240(2):205-213. https://doi.org/10.1097/01.sla.0000133083.54934.ae

8. Bittner JGt, Alrefai S, Vy M, Mabe M, Del Prado PAR, Clingempeel NL (2018) Comparative analysis of open and robotic transversus abdominis release for ventral hernia repair. Surg Endosc 32(2):727-734. https://doi.org/10.1007/s00464-017-5729-0

9. Slankamenac K, Graf R, Barkun J, Puhan MA, Clavien PA (2013) The comprehensive complication index: a novel continuous scale to measure surgical morbidity. Ann Surg 258(1):1-7. https://doi. org/10.1097/SLA.0b013e318296c732

10. Morales-Conde S (2012) A new classification for seroma after laparoscopic ventral hernia repair. Hernia 16(3):261-267. https ://doi.org/10.1007/s10029-012-0911-8

11. Gokcal F, Kudsi Y (2019) Transabdominal Preperitoneal (rTAPP) Repair. In: Robotic assisted hernia repair. Springer, pp 287-301

12. Khan N, Abboudi H, Khan MS, Dasgupta P, Ahmed K (2014) Measuring the surgical 'learning curve': methods, variables and competency. BJU Int 113(3):504-508. https://doi.org/10.1111/ bju. 12197

13. Mackenzie H, Miskovic D, Ni M, Parvaiz A, Acheson AG, Jenkins JT, Griffith J, Coleman MG, Hanna GB (2013) Clinical and educational proficiency gain of supervised laparoscopic colorectal surgical trainees. Surg Endosc 27(8):2704-2711. https://doi. org/10.1007/s00464-013-2806-x

14. Waldman JD, Yourstone SA, Smith HL (2003) Learning curves in health care. Health Care Manag Rev 28(1):41-54. https://doi. org/10.1097/00004010-200301000-00006

15. Bencini L, Sanchez LJ (2004) Learning curve for laparoscopic ventral hernia repair. Am J Surg 187(3):378-382. https://doi. org/10.1016/j.amjsurg.2003.12.005

16. Salameh JR, Sweeney JF, Graviss EA, Essien FA, Williams MD, Awad S, Itani KM, Fisher WE (2002) Laparoscopic ventral hernia repair during the learning curve. Hernia 6(4):182-187. https://doi. org/10.1007/s10029-002-0083-Z

17. Schreuder HW, Wolswijk R, Zweemer RP, Schijven MP, Verheijen RH (2012) Training and learning robotic surgery, time for a more structured approach: a systematic review. BJOG 119(2):137-149. https://doi.org/10.1111/j.1471-0528.2011.03139.x

18. Muysoms F, Van Cleven S, Pletinckx P, Ballecer C, Ramaswamy A (2018) Robotic transabdominal retromuscular umbilical prosthetic hernia repair (TARUP): observational study on the operative time during the learning curve. Hernia 22(6):1101-1111. https:// doi.org/10.1007/s10029-018-1825-x

19. Tulloh B, de Beaux A (2016) Defects and donuts: the importance of the mesh:defect area ratio. Hernia 20(6):893-895. https://doi. org/10.1007/s 10029-016-1524-4

20. Flament JB, Avisse C, Delattre JF (2001) Anatomy of the abdominal wall. In: Abdominal wall hernias. Springer, pp 39-63

21. Fraser SA, Feldman LS, Stanbridge D, Fried GM (2005) Characterizing the learning curve for a basic laparoscopic drill. Surg Endosc Other Interv Tech 19(12):1572-1578. https://doi. org/10.1007/s00464-005-0150-5

Publisher's Note Springer Nature remains neutral with regard to jurisdictional claims in published maps and institutional affiliations. 